\title{
MiR-301a Promotes Colorectal Cancer Cell Growth and Invasion by Directly Targeting SOCS6
}

\author{
Yantian Fang Bo Sun Jianbin Xiang Zongyou Chen \\ Department of General Surgery, Huashan Hospital affiliated to Fudan University, Shanghai P. R. China
}

\section{Key Words}

MiR-301a • Colorectal cancer • SOCS6 $•$ Proliferation • Migration • Invasion

\begin{abstract}
Background/Aims: Colorectal cancer (CRC) is one of the most common malignancies worldwide, and microRNAs play a crucial role in CRC biology. The purpose of this study was to investigate the exact functions and potential mechanisms of action of miR-301a in CRC. Methods: Quantitative real-time PCR was conducted to assess the expression of miR-301a. Cell proliferation was detected using MTT and colony formation assay, and cell invasion and migration were evaluated using Transwell assay. Luciferase reporter assay was used to identify the direct regulation of suppressor of cytokine signaling 6 (SOCS6) by miR-301a. Results: We first confirmed the upregulation of miR-301a in CRC tissues and cell lines. Gain-of-function and loss-of-function studies in the human CRC cell lines, SW480 and SW620, showed that miR-301a acts as an oncogene by increasing cell proliferation, migration and invasion as well as tumor growth. Furthermore, SOCS6 was identified as a target gene of miR-301a. Reintroduction of SOCS6 partially abrogated miR-301a-induced cell proliferation, migration and invasion. Conclusion: These data suggest that miR-301a promotes CRC progression by directly downregulating SOCS6 expression, and miR-301a may represent a novel biomarker for the prevention and treatment of CRC.
\end{abstract}

J. Xiang and

Z. Chen

\section{KARGER 125}

Department of General Surgery, Huashan Hospital, No.3 building 6th floor, No.12 Wulumuqizhong Rd, Jin'an District, Shanghai 200040 (P.R. China)

Fax:+86 021 52887224, E-Mail xjbzhw@163.com E-Mail czyshhs1@126.com 


\section{Introduction}

Colorectal cancer (CRC) is one of the most common malignant tumors worldwide [1]. Disease progression and metastases are responsible for the majority of cancer deaths, and metastatic disease is present in up to $30 \%$ of cases at presentation [2-4]. Although major efforts have been made in metastasis research in recent decades, patients with advanced CRC continue to experience poor prognosis and a high death rate $[5,6]$. Consequently, identification of potential targets for early diagnosis and therapy of CRC is urgently required.

MicroRNAs (miRNAs) are small ( 21-25 nucleotides), non-coding single stranded RNAs that are a novel cluster of target gene regulators [7]. These small RNAs are involved in various biological processes associated with cancer, such as cell proliferation, apoptosis, differentiation and invasion, acting by repressing translation of mRNA to protein or inducing mRNA cleavage in a sequence-specific manner [8]. Accumulating evidence has demonstrated that miRNAs contribute to CRC tumorigenesis and progression as oncogenes or tumor suppressors [9-11]. For example, miR-198 was reported to be down-regulated in CRC tissues and to repress tumor growth and metastasis by targeting fucosyl transferase 8 [12]. Fang et al. found that overexpression of miR-17-5p promoted chemotherapeutic drug resistance and tumor metastasis of colorectal cancer by repressing PTEN expression [13]. Park et al. showed that miR-124, miR-27a, and miR-30b negatively regulated KITENIN expression and that through this mechanism they suppressed the migration and invasion of several CRC cell lines [14]. Therefore, miRNAs could serve as therapeutic targets in CRC. A recent report demonstrated that expression levels of miR-301a are significantly upregulated in CRC [15]. However, the potential mechanism of miR-301a in CRC has not been completely elucidated.

In this study, we first confirmed that expression levels of miR-301a were upregulated in CRC tissues and cell lines. Further study showed that miR-301a promoted CRC cell proliferation, migration, and invasion in vitro and tumor growth in vivo. The oncogenic effects of miR-301a could be partially attributed to the decreased expression of suppressor of cytokine signaling 6 (SOCS6). These results provide new insights into the potential mechanisms of CRC oncogenesis and metastasis.

\section{Materials and Methods}

Tissue samples and cell lines

Twenty pairs of CRC tissues and matched adjacent normal tissues were obtained from patients in Huashan Hospital, with written informed consent and agreement. This study was approved by the Medical Ethics Committee of Huashan Hospital. All specimens were from untreated patients undergoing surgery and were snap-frozen and preserved at $-80^{\circ} \mathrm{C}$. Human CRC cell lines (SW480, HT29, SW620 and LoVo) were purchased from the cell bank of the Chinese Academy of Sciences (Shanghai, China) and cultured in DMEM (Invitrogen, Carlsbad, CA) supplemented with 10\% fetal bovine serum (Invitrogen). A normal colon epithelial cell line (FHC) was grown in DMEM: F12 supplemented with 10\% FBS.

\section{Lentivirus production, plasmid construction and transduction}

Expression plasmids of miR-301a, antisense oligonucleotides against miR-301a (anti-miR-301a), and the SOCS6 coding region with and without the 3'UTR were purchased from Hanbio (Shanghai, China). Lentiviral packaging and infection of CRC cells were performed according to the manufacturer's protocol (Hanbio). The $400 \mathrm{bp}$ sequence of the SOCS6 3'-UTR containing the predicted miR-301a binding sites was amplified by PCR and cloned into pMIR-REPORT (Ambion, Austin, TX) according to the manufacturer's instructions. A mutant miR-301a binding site with three mismatched nucleotides was created using the QuikChange site-directed mutagenesis kit (Stratagene, Santa Clara, CA) and confirmed by sequencing.

RNA extraction and quantitative real-time RT-PCR

Total RNA was extracted with TRIzol reagent (Invitrogen) and treated with RNase-free DNase I (Qiagen, Hilden, Germany). For miRNA expression analysis, $1 \mu \mathrm{g}$ of DNase I-treated RNA was converted into cDNA 
Fang et al:: MiR-301a Exerts its Oncogenic Effect in CRC

using the TaqMan microRNA reverse transcription kit (Applied Biosystems, Foster City, CA). Expression levels of mature miR-301a were measured using a TaqMan microRNA assay (Applied Biosystems) by normalizing to U6 snRNA. For analysis of SOCS6 transcript levels, $1 \mu \mathrm{g}$ of DNase I-treated RNA was reverse transcribed using the SuperScript III first-strand synthesis system supermix (Invitrogen). A SYBR Green PCR kit (TaKaRa, Dalian, China) was used to quantify the SOCS6 mRNA levels. $\beta$-actin mRNA was amplified as an internal control. Expression levels of miR-301a or SOCS6 were calculated using the 2- $\Delta \Delta \mathrm{CT}$ method.

\section{Cell proliferation and colony formation assays}

Cell proliferation was measured using the MTT assay. Cells $\left(1.5 \times 10^{3}\right)$ were seeded into 96 -well plates, and $20 \mu \mathrm{l}$ of $5 \mathrm{mg} / \mathrm{ml} \mathrm{MTT}$ was added to each well at $0,24,48,72$, and $96 \mathrm{~h}$. The cells were then incubated at $37^{\circ} \mathrm{C}$ for another $4 \mathrm{~h}$ before $150 \mu \mathrm{l}$ DMSO was added. Cell viability was assessed by measurement of absorbance at $490 \mathrm{~nm}$ using a microplate reader. For colony formation assay, cells (200/well) were plated into six-well plates and cultured for 14 days. At the end of the culture period, the cells were stained with $0.05 \%$ crystal violet for at least 1 hour. The number of colonies was assessed in four different fields. Experiments were conducted in triplicate.

\section{Wound healing and matrigel invasion assays}

For the wound healing assay, cells $(5 \times 105)$ were seeded into six-well plates and cultured under standard conditions. When the cells reached confluence, a wound was made by scraping the cell monolayer with a $200 \mu \mathrm{l}$ pipette tip. Cell migration was determined by measuring the movement of cells into the scraped area. The process of wound closure was observed and photographed after $24 \mathrm{~h}$. For the cell invasion assay, cells $(1 \times 105)$ were resuspended in serum-free medium and then plated into a transwell chamber. The chambers were incubated for $48 \mathrm{~h}$ with complete culture medium added to the lower chamber. Nonmigrated cells were removed and chambers were stained with crystal violet. Photomicrographs of five regions were captured from duplicate chambers and the numbers of cells were counted. Experiments were conducted in triplicate.

\section{Dual luciferase reporter assay}

SW480 cells were seeded into 24-well plates and cotransfected the next day with pMIR-REPORT-3'UTR SOCS6 or pMIR-REPORT-3'-UTR-mut SOCS6, pMIR-Renilla expressing vector and hsa-miR-301a vector using Lipofectamine 2000 (Invitrogen). At $48 \mathrm{~h}$ post-transfection, cells were lysed and analyzed by luciferase reporter assay using the Dual-Luciferase Reporter Assay System (Promega, Madison, WI). Experiments were conducted in triplicate.

\section{Western blot analysis}

Western blot analysis was carried out as previously described [16]. Briefly, cells were lysed in RIPA buffer containing a protease and phosphatase inhibitor mixture (Roche, Indianapolis, IN). Equal amounts of protein were resolved by SDS-PAGE, transferred onto polyvinylidene fluoride (PVDF) membranes (Millipore, Bedford, MA) and then detected by immunoblotting using an enhanced chemi-luminescence detection system. The antibody against SOCS6 was purchased from Abcam (Abcam, Cambridge, MA). The $\beta$-actin antibody was obtained from Santa Cruz Biotechnology.

\section{In vivo tumorigenesis assay}

Female BALB/c nude mice (4-6 weeks old) were injected subcutaneously in both flanks with $1 \times 106$ SW480 cells stably expressing miR-301a or empty vector controls. Tumor width (a) and length (b) were measured every 5 days using calipers and tumor volume was calculated by the formula $V=a \times b 2 / 2$. The mice were sacrificed and photographed after 5 weeks. All animal experiments were approved by the Animal Care and Use Committee of Fudan University.

\section{Immunohistochemistry}

SOCS6 was detected in paraffin-embedded CRC tumor tissues and adjacent normal tissues. Immunohistochemical staining was performed as described previously [17].

\section{KARGER}


Statistical Analysis

All results are expressed as mean \pm SD from three independent experiments and differences were compared using Student's two-tailed t test. Correlations were analyzed by Spearman's correlation analysis. $\mathrm{P}<0.05$ was considered significant.

\section{Results}

Upregulated miR-301a expression in CRC tissues and cell lines

To understand the role of miR-301a in CRC, we examined levels of miR-301a expression by qRT-PCR in 20 pairs of CRC tissues and their adjacent normal tissues. As shown in Fig. $1 \mathrm{~A}$, the expression of miR-301a was significantly increased in CRC tissues compared with paired adjacent normal tissues. We further assessed the expression levels of miR-301a in CRC cell lines (SW480, LOVO, SW620 and HT29) and normal colon epithelial cell lines (FHC). Consistent with the results found in CRC tissues, miR-301a levels were significantly higher in all four CRC cell lines tested compared with FHC (Fig. 1B), suggesting that miR-301a is upregulated in CRC.

MiR-301a promotes CRC cell growth in vitro and in vivo

Given that miR-301a was significantly upregulated in CRC tissues and cell lines, we investigated whether miR-301a plays an oncogenic role in CRC development. SW480 cells were used to do the rescue experiment because these cells have low endogenous miR-301a expression in CRC cell lines, while SW620 cells were used to do the knockdown experiment because they have high endogenous miR-301a expression. Overexpression or inhibition of mature miR-301a was confirmed by qRT-PCR (Fig. 2A). MTT assay showed that introduction of miR-301a was able to significantly promote SW480 cell proliferation, whereas miR-301a inhibition caused SW620 cells to proliferate more slowly than the negative control (Fig. 2B). Similarly, overexpression of miR-301a significantly increased the ability of SW480 cells to form colonies (Fig. 2C). To determine the potential roles of miR-301a in tumor growth in vivo, we injected nude mice with miR-con-infected or miR-301a-infected SW480 cells. Tumor growth was monitored regularly. The results showed that the miR-301a overexpression group exhibited a markedly faster growth rate (Fig. 2D). Both tumor volume and weight from the miR-301a overexpression group were significantly increased compared with the miRcon group (Fig. 2E and F). Collectively, these results suggest that miR-301a has an oncogenic function in CRC.

MiR-301a promotes CRC cell migration and invasion

To further verify the oncogenic function of miR-301a in CRC, we next evaluated the effect of miR-301a on the migration and invasion capacity of CRC cells using the woundhealing assay and the matrigel invasion assay. As shown in Fig. 3A and B, overexpression of miR-301a significantly enhanced SW480 cell migration and invasion capabilities compared with the negative control, whereas miR-301a inhibition caused SW620 cells to move more slowly than the negative control, demonstrating the oncogenic effects of miR-301a on the migration and invasion of CRC cells.

\section{MiR-301a directly targets SOCS6}

We next searched for the target genes of miR-301a using publicly available databases (TargetScan, Pictar and miRANDA). SOCS6 was selected as a potential downstream target of miR-301a based on the putative target sites at 21-27 base pairs of the SOCS6 3'UTR (Fig. 4A). To confirm the target sites, the wild-type 3'-UTR region of the SOCS6 gene containing a putative binding site of miR-301a and a mutant $3^{\prime} \mathrm{UTR}$ were amplified and inserted into a luciferase reporter vector. The luciferase activity assay showed that miR-301a significantly suppressed luciferase activity in the wild type but not the mutant 3'UTR of SOCS6 (Fig. 4B). Furthermore, ectopic expression of miR-301a resulted in a significant reduction in SOCS6 


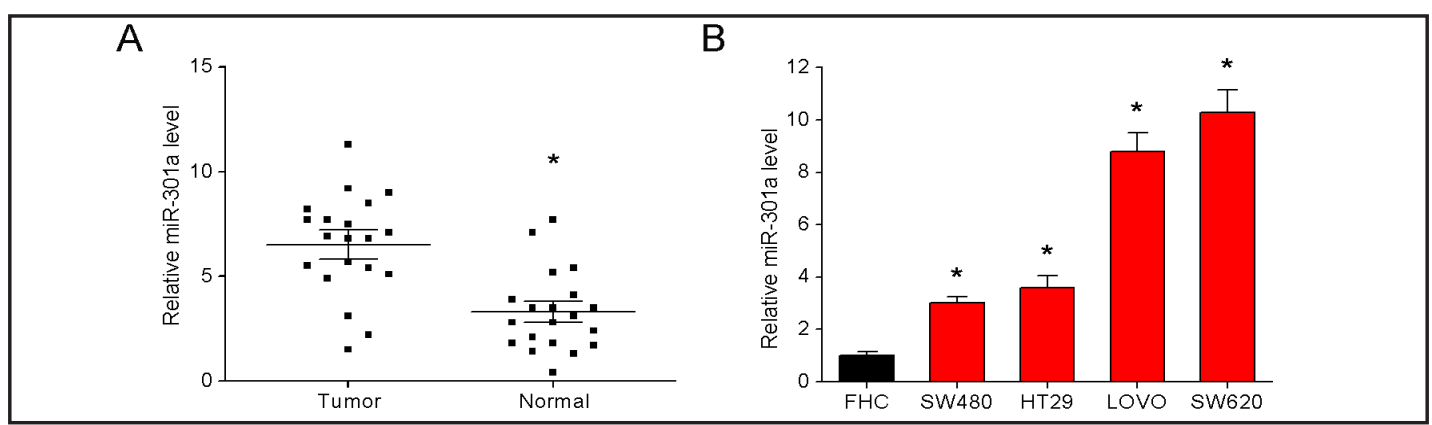

Fig. 1. Expression of miR-301a is upregulated in CRC tissues and cell lines. (A) Expression levels of miR301a were examined by qRT-PCR in CRC tissues and paired adjacent normal tissues of twenty patients. (B) Expression levels of miR-301a in four human CRC cell lines and normal colon epithelial cell lines (FHC). Transcript levels were normalized to U6 expression. ${ }^{*} P<0.05$.

A

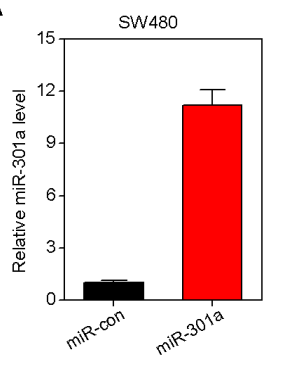

C

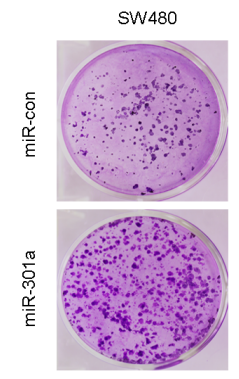

D

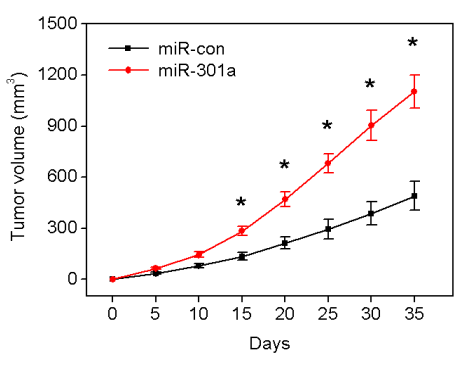

B
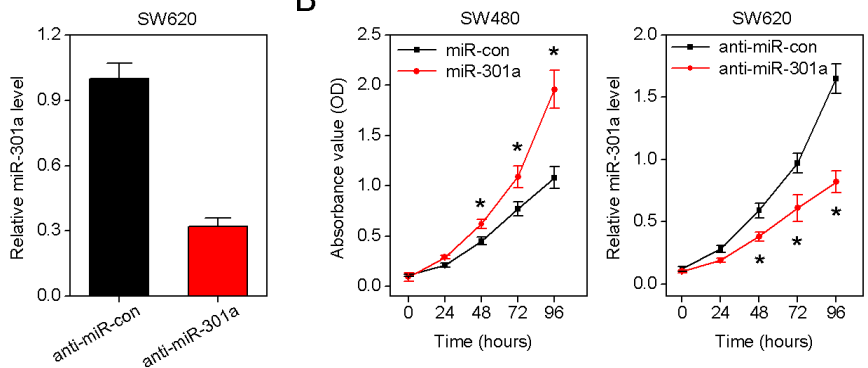

SW620
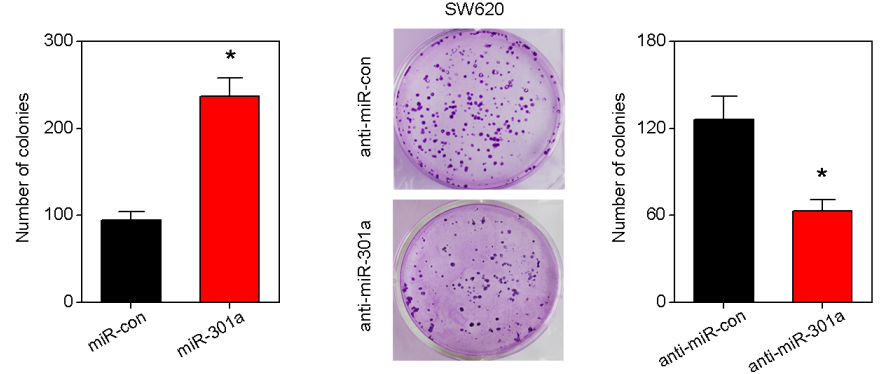

F

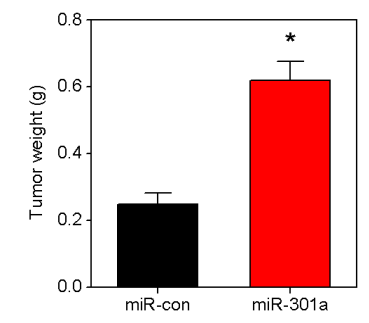

Fig. 2. miR-301a promotes CRC cell growth in vitro and in vivo. (A) Relative expression levels of miR-301a in SW480 and SW620 cells after transduction with lentivirus encoding miR-301a or anti-miR-301a. (B) The effect of miR-301a on cell proliferation was measured by MTT assay. (C) Representative micrographs and quantification of crystal violet-stained cell colonies. (D) miR-con-infected and miR-301a-infected SW480 cells were injected subcutaneously into the flank of nude mice. Tumor volumes were measured every 5 days. (E) Photographs showing representative tumors formed. (F) Tumor weight was calculated at 5 weeks post injection. ${ }^{*} P<0.05$. 


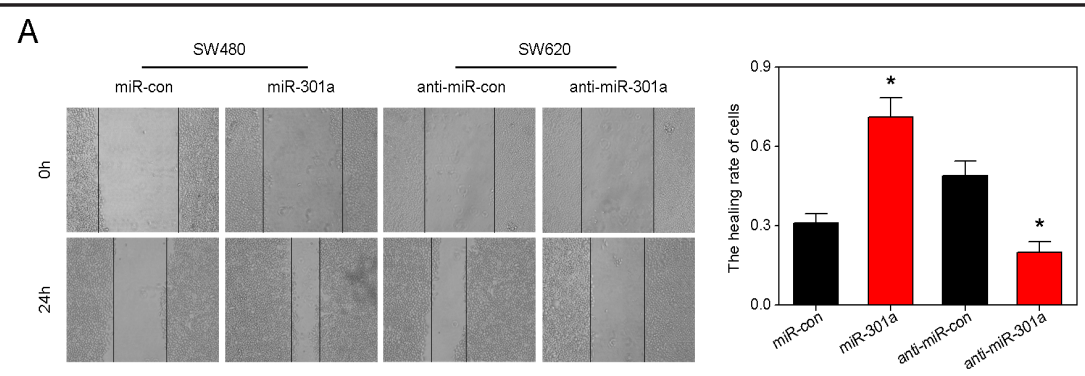

B

B sw480
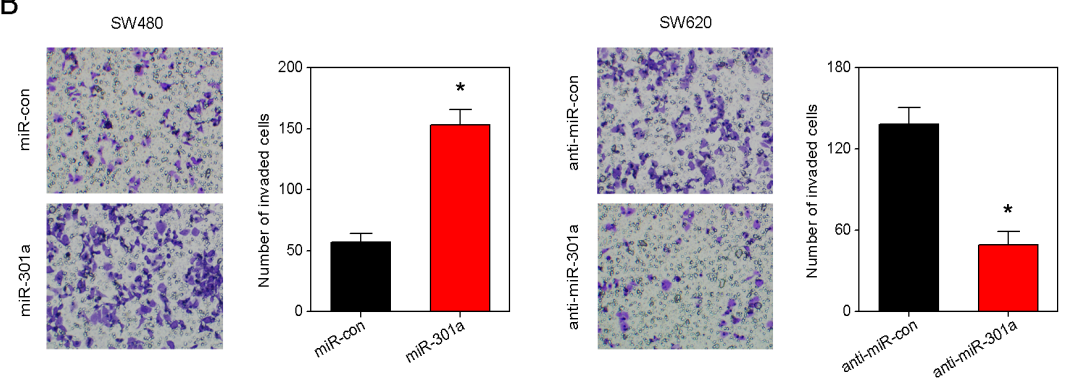

Fig. 3. miR-301a promotes CRC cell migration and invasion. (A) The wound healing assay showed the migration ability of CRC cells transduced with lentivirus encoding miR-301a or anti-miR-301a. (B) The transwell assay showed that miR-301a significantly promoted cell invasion, while anti-miR-301a suppressed cell invasion. ${ }^{*} P<0.05$.

A

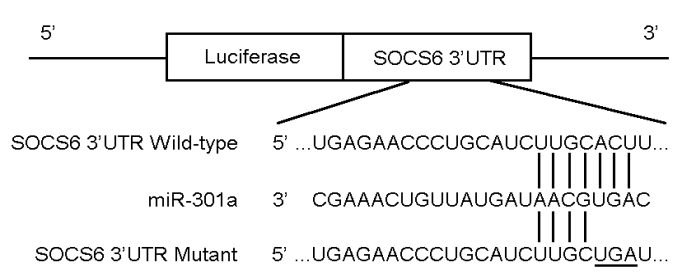

C
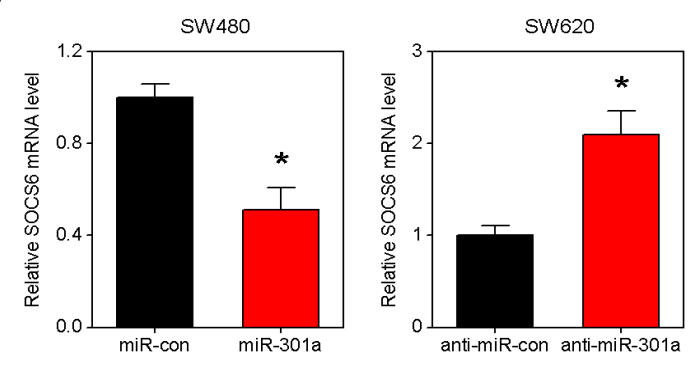

B

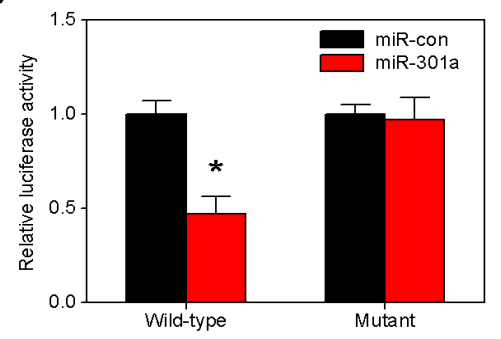

D

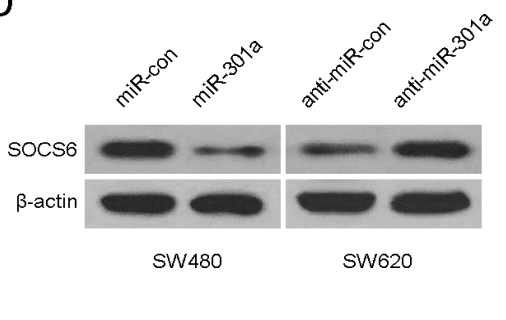

Fig. 4. SOCS6 is a direct target of miR-301a. (A) Predicted miR-301a target sequence in the $3^{\prime}$-UTR of the SOCS6 gene. A mutation was generated in the seed region of the SOCS6 3'UTR as indicated by the underline. (B) The reporter plasmids containing the wild type $3^{\prime}$-UTR of the SOCS6 gene or mutant were co-transfected into SW480 cells with miR-301a expressing plasmid to evaluate luciferase activity. (C) Expression of SOCS6 mRNA in the indicated cells was analyzed by qRT-PCR. (D) Protein levels of SOCS6 in the indicated cells were examined by western blot. ${ }^{*} \mathrm{P}<0.05$.

mRNA and protein expression, evaluated by qRT-PCR and western blotting (Fig. 4C and D). In contrast, inhibition of miR-301a led to the upregulation of SOCS6 mRNA and protein. Next, we detected SOCS6 in CRC tissues and adjacent normal tissues. Immunohistochemical 
Fig. 5. SOCS6 is negatively correlated with miR-301a in CRC tissues. (A) Immunohistochemical staining for SOCS6 in CRC (left) and adjacent normal tissues (right), magnification $\times 100$. (B) The expression levels of SOCS6 were examined by qRT-PCR in CRC tissues and paired adjacent normal tissues of twenty patients. (C) Correlation between miR-301a and SOCS6 mRNA expression in CRC tissues. (Spearman's correlation analysis, $r=-0.676$, $P=0.001)$.

Fig. 6. SOCS6 contributes to miR-301a-induced proliferation, migration and invasion of CRC cells. (A) Exogenous expression of SOCS6 was performed by transducing the lentivirus encoding SOCS6 with or without the 3'UTR into SW480/ miR-301a cells. The MTT assay was used to examine proliferation. (B) Quantification of crystal violet-stained cell colonies of the indicated cells. (C) Migration ability was detected by the wound healing assay. (D) Invasion ability was examined by the matrigel invasion assay. ${ }^{*} P<0.05$ vs. miR301 a group.

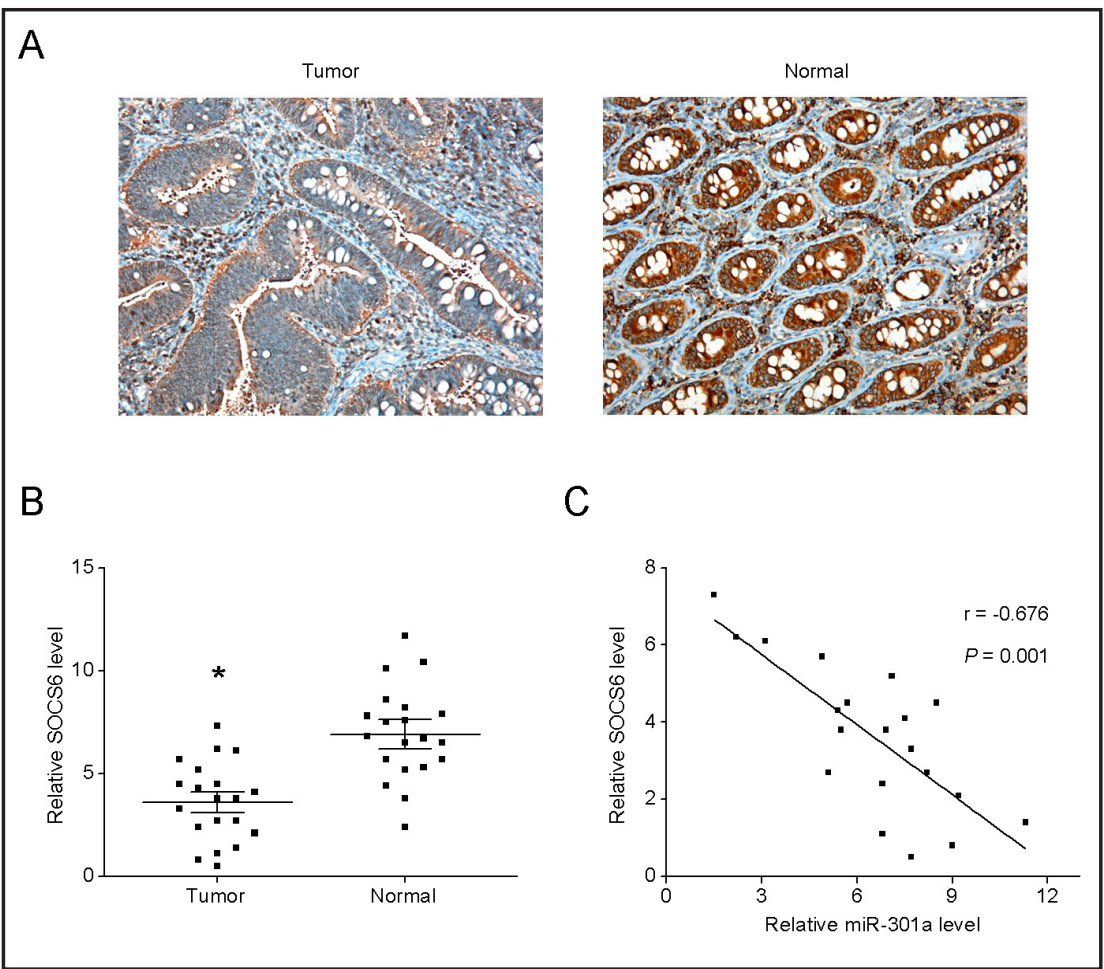

A

B

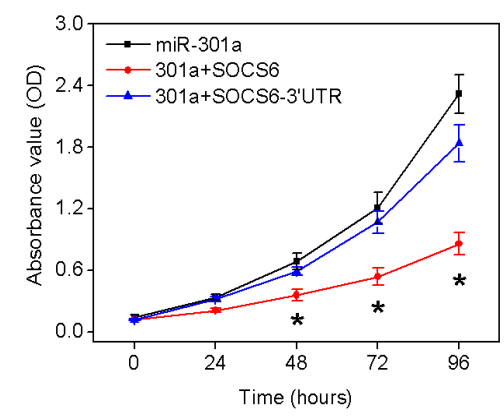

C

D
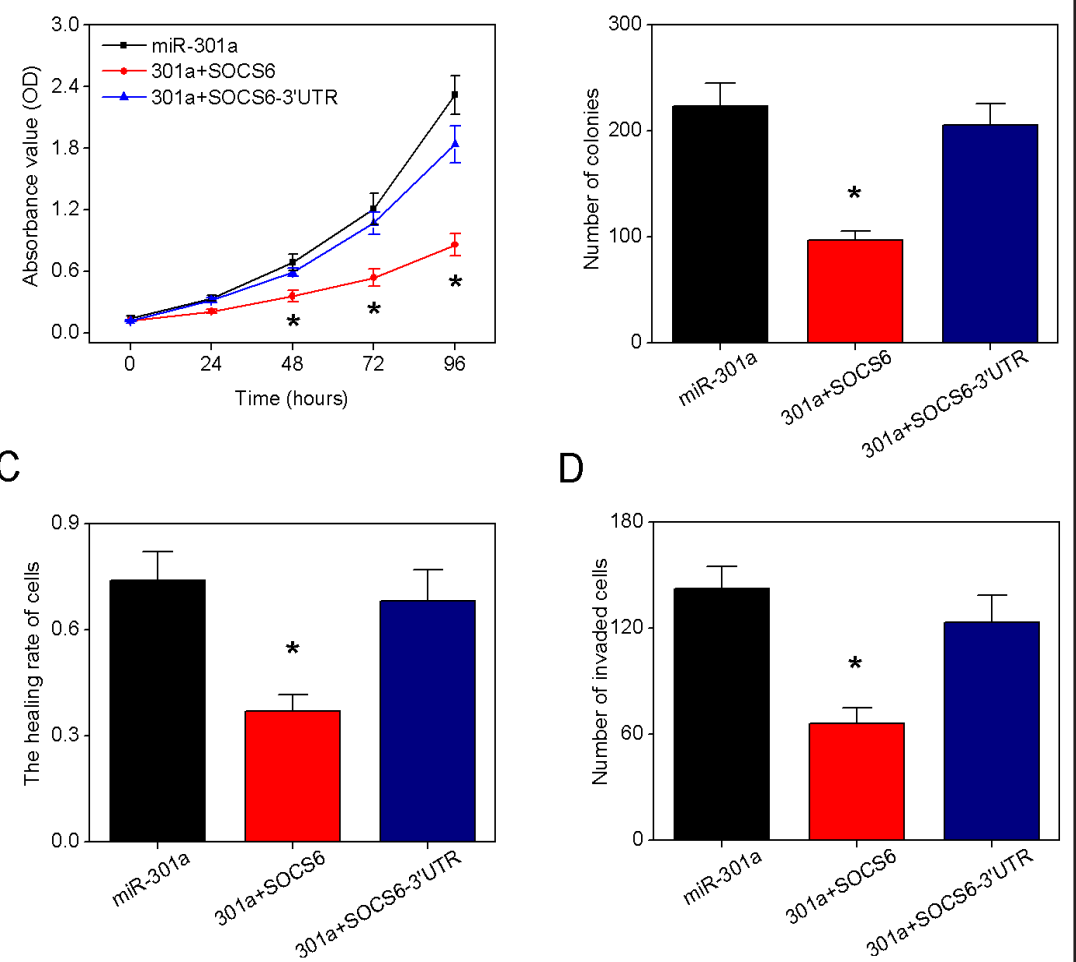

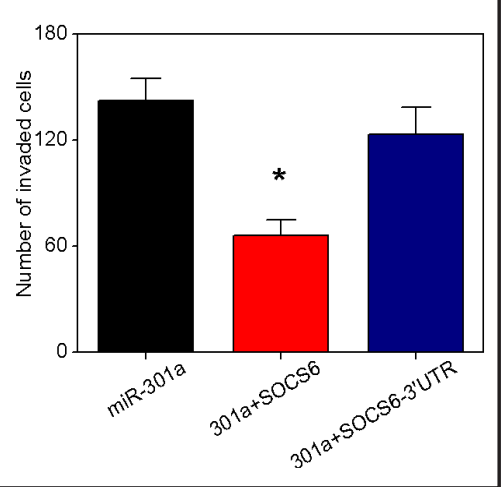

staining and qRT-PCR analysis showed that SOCS6 expression was markedly reduced in CRC tissues compared with the corresponding normal tissues (Fig. 5A and B). Statistical analysis demonstrated that the level of miR-301a was inversely correlated with the level of SOCS6 
mRNA in human CRC tissues ( $\mathrm{r}=-0.676, P=0.001$; Fig. $5 \mathrm{C}$ ). Taken together, these results suggest that SOCS6 is a genuine downstream target of miR-301a.

Overexpression of SOCS6 impaired the oncogenic effects of miR-301a

To investigate whether the regulatory effects of miR-301a on the proliferation, migration and invasion of CRC cells are mediated by SOCS6, lentivirus encoding SOCS6 (without $3^{\prime}$-UTR) or SOCS6-3'-UTR (with 3'-UTR) was transduced into miR-301a-overexpressing SW480 cells. As expected, reintroduction of SOCS6 could attenuate miR-301a-induced cell proliferation, migration and invasion, whereas expression of SOCS6-3'-UTR could not (Fig. 6A-D). Thus, these data suggest that SOCS6 restoration partially counteracts the oncogenic functions of miR-301a.

\section{Discussion}

The abnormal expression of miRNAs is commonly observed in human tumors and it has been shown that miRNAs can be tumorigenic, acting as tumor suppressors or oncogenes [18-20]. Recent evidence has shown that aberrant miRNA expression plays important roles in CRC development and progression [9]. However, knowledge of the abnormal expression and potential role of miRNAs in CRC is largely lacking. Here, we investigated the biological and pathological roles of miR-301a in CRC.

Upregulation of miR-301a has been reported in many human cancers, including gastric cancer, pancreatic cancer, hepatocellular carcinoma (HCC), and breast cancer. Wang et al. showed that miR-301a was significantly upregulated in gastric cancer and promoted cell growth, soft agar clonogenicity, migration, invasion, and decreased cell apoptosis [21]. Chen et al. found that miR-301a overexpression promoted pancreatic cancer cell proliferation by directly inhibiting Bim expression [22]. miR-301a was frequently upregulated in HCC and shown to play an important role in increasing proliferation, migration and invasion and in inhibiting apoptosis of HCC cells [23]. Upregulated microRNA-301a was also reported to promote metastasis in breast cancer by targeting PTEN and activating Wnt/ $\beta$-catenin signaling [24]. Taken together, these findings support an important role of altered miR-301a during tumor development and progression.

It has been shown that miR-301a expression is significantly upregulated in CRC tissues compared with adjacent normal tissues [15]. However, the biological functions of miR-301a in CRC are still unclear and remain to be further elucidated. In the present study, we first confirmed that miR-301a expression is increased in CRC tissues and cell lines. By gainof-function and loss-of-function studies, we demonstrated that miR-301a promotes cell proliferation, migration and invasion in vitro as well as tumor growth in vivo. Furthermore, we identified SOCS6 as a direct target gene of miR-301a. The luciferase activity assay showed that miR-301a can bind the 3'-UTR of the SOCS6 transcript. Overexpression of miR-301a significantly downregulated the expression of SOCS6 mRNA and protein in SW480 cells, whereas inhibition of miR-301a significantly upregulated the expression of SOCS6 mRNA and protein in SW620 cells. Moreover, we observed low SOCS6 expression and significant inverse correlation of miR-301a and SOCS6 in CRC tissues. Thus, our data suggest that miR301a targets SOCS6 and downregulates its expression in CRC.

Suppressor of cytokine signaling 6 (SOCS6) is a member of the SOCS family and is mainly involved in negative regulation of receptor tyrosine kinase signaling. Reduced expression of SOCS6 has been observed in many human cancers and its down-regulation is correlated with aggressive tumor progression and poor prognosis, suggesting the importance of SOCS6 in tumor growth and progression [25-28]. It was recently reported that the SOCS6 level is also reduced in CRC and may serve as a diagnostic biomarker for CRC patients [17]. This result is consistent with our findings that SOCS6 is a functional target of miR-301a. To specifically address the implication of SOCS6 in the oncogenic roles of miR-301a, lentivirus coding SOCS6 (without 3'-UTR) or SOCS6-3'-UTR (with 3'-UTR) was transduced into miR-301a- 
overexpressing SW480 cells. Our results show that upregulation of SOCS6 (without $3^{\prime}$ UTR) could significantly reverse the effect of miR-301a in SW480 cells. These results suggest that miR-301a regulates CRC cell proliferation, migration and invasion by targeting SOCS6.

In summary, our studies reveal that miR-301a acts as an oncogene in CRC and that SOCS6 is a direct downstream target of miR-301a and can be involved in its multiple oncogenic effects. Thus, inhibition of miR-301a may be a promising molecular therapy in CRC treatment.

\section{Acknowledgments}

This work was supported by a Grant from Shanghai Science and Technology Department of Medicine guiding biomedical projects (No. 134119a1400).

\section{References}

1 Boyle P, Ferlay J: Cancer incidence and mortality in europe, 2004. Ann Oncol 2005;16:481-488.

2 Zhao H, Dong T, Zhou H, Wang L, Huang A, Feng B, Quan Y, Jin R, Zhang W, Sun J, Zhang D, Zheng M: Mir320a suppresses colorectal cancer progression by targeting rac1. Carcinogenesis 2014;35:886-895.

3 Weitz J, Koch M, Debus J, Hohler T, Galle PR, Buchler MW: Colorectal cancer. Lancet 2005;365:153-165.

4 Wang B, Li W, Liu H, Yang L, Liao Q, Cui S, Wang H, Zhao L: Mir-29b suppresses tumor growth and metastasis in colorectal cancer via downregulating tiam1 expression and inhibiting epithelialmesenchymal transition. Cell Death Dis 2014;5:e1335.

5 Manfredi S, Lepage C, Hatem C, Coatmeur O, Faivre J, Bouvier AM: Epidemiology and management of liver metastases from colorectal cancer. Ann Surg 2006;244:254-259.

6 Yang MH, Yu J, Jiang DM, Li WL, Wang S, Ding YQ: Microrna-182 targets special at-rich sequence-binding protein 2 to promote colorectal cancer proliferation and metastasis. J Transl Med 2014;12:109.

7 Gong H, Liu CM, Liu DP, Liang CC: The role of small rnas in human diseases: Potential troublemaker and therapeutic tools. Med Res Rev 2005;25:361-381.

-8 Bartel DP: Micrornas: Target recognition and regulatory functions. Cell 2009;136:215-233.

-9 Lanza G, Ferracin M, Gafa R, Veronese A, Spizzo R, Pichiorri F, Liu CG, Calin GA, Croce CM, Negrini M: Mrna/ microrna gene expression profile in microsatellite unstable colorectal cancer. Mol Cancer 2007;6:54.

10 Link A, Balaguer F, Shen Y, Nagasaka T, Lozano JJ, Boland CR, Goel A: Fecal micrornas as novel biomarkers for colon cancer screening. Cancer Epidemiol Biomarkers Prev 2010;19:1766-1774.

11 Asangani IA, Rasheed SA, Nikolova DA, Leupold JH, Colburn NH, Post S, Allgayer H: Microrna-21 (mir-21) post-transcriptionally downregulates tumor suppressor pdcd 4 and stimulates invasion, intravasation and metastasis in colorectal cancer. Oncogene 2008;27:2128-2136.

12 Wang M, Wang J, Kong X, Chen H, Wang Y, Qin M, Lin Y, Xu J, Hong J, Chen YX, Zou W, Fang JY: Mir-198 represses tumor growth and metastasis in colorectal cancer by targeting fucosyl transferase 8. Sci Rep 2014;4:6145.

13 Fang L, Li H, Wang L, Hu J, Jin T, Wang J, Yang BB: Microrna-17-5p promotes chemotherapeutic drug resistance and tumour metastasis of colorectal cancer by repressing pten expression. Oncotarget 2014;5:2974-2987.

14 Park SY, Kim H, Yoon S, Bae JA, Choi SY, Jung YD, Kim KK: Kitenin-targeting microrna-124 suppresses colorectal cancer cell motility and tumorigenesis. Mol Ther 2014;22:1653-1664.

15 Huang Z, Huang S, Wang Q Liang L, Ni S, Wang L, Sheng W, He X, Du X: Microrna-95 promotes cell proliferation and targets sorting nexin 1 in human colorectal carcinoma. Cancer Res 2011;71:2582-2589.

16 Weng H, Huang H, Dong B, Zhao P, Zhou H, Qu L: Inhibition of mir-17 and mir-20a by oridonin triggers apoptosis and reverses chemoresistance by derepressing bim-s. Cancer Res 2014;74:4409-4419.

17 Letellier E, Schmitz M, Baig K, Beaume N, Schwartz C, Frasquilho S, Antunes L, Marcon N, Nazarov PV, Vallar L, Even J, Haan S: Identification of socs2 and socs6 as biomarkers in human colorectal cancer. Br J Cancer 2014;111:726-735. 
Fang et al.: MiR-301a Exerts its Oncogenic Effect in CRC

18 Olson P, Lu J, Zhang H, Shai A, Chun MG, Wang Y, Libutti SK, Nakakura EK, Golub TR, Hanahan D: Microrna dynamics in the stages of tumorigenesis correlate with hallmark capabilities of cancer. Genes Dev 2009;23:2152-2165.

19 Esquela-Kerscher A, Slack FJ: Oncomirs - micrornas with a role in cancer. Nat Rev Cancer 2006;6:259-269.

20 Calin GA, Croce CM: Microrna signatures in human cancers. Nat Rev Cancer 2006;6:857-866.

21 Wang M, Li C, Yu B, Su L, Li J, Ju J, Yu Y, Gu Q, Zhu Z, Liu B: Overexpressed mir-301a promotes cell proliferation and invasion by targeting runx3 in gastric cancer. J Gastroenterol 2013;48:1023-1033.

22 Chen Z, Chen LY, Dai HY, Wang P, Gao S, Wang K: Mir-301a promotes pancreatic cancer cell proliferation by directly inhibiting bim expression. J Cell Biochem 2012;113:3229-3235.

23 Zhou P, Jiang W, Wu L, Chang R, Wu K, Wang Z: Mir-301a is a candidate oncogene that targets the homeobox gene gax in human hepatocellular carcinoma. Dig Dis Sci 2012;57:1171-1180.

24 Ma F, Zhang J, Zhong L, Wang L, Liu Y, Wang Y, Peng L, Guo B: Upregulated microrna-301a in breast cancer promotes tumor metastasis by targeting pten and activating wnt/beta-catenin signaling. Gene 2014;535:191-197.

25 Wu Q, Luo G, Yang Z, Zhu F, An Y, Shi Y, Fan D: Mir-17-5p promotes proliferation by targeting socs6 in gastric cancer cells. FEBS Lett 2014;588:2055-2062.

26 Zhu JG, Dai QS, Han ZD, He HC, Mo RJ, Chen G, Chen YF, Wu YD, Yang SB, Jiang FN, Chen WH, Sun ZL, Zhong WD: Expression of socss in human prostate cancer and their association in prognosis. Mol Cell Biochem 2013;381:51-59.

27 Qiu X, Zheng J, Guo X, Gao X, Liu H, Tu Y, Zhang Y: Reduced expression of socs2 and socs6 in hepatocellular carcinoma correlates with aggressive tumor progression and poor prognosis. Mol Cell Biochem 2013;378:99-106.

28 Sriram KB, Larsen JE, Savarimuthu Francis SM, Wright CM, Clarke BE, Duhig EE, Brown KM, Hayward NK, Yang IA, Bowman RV, Fong KM: Array-comparative genomic hybridization reveals loss of socs6 is associated with poor prognosis in primary lung squamous cell carcinoma. PLoS One 2012;7:e30398. 